\title{
Galileo's Problem with PRS or What's in a Phase?
}

\author{
M. Stephen Hodgart \\ Surrey Space Centre, University of Surrey, Guildford, Surrey GU27XH, UK \\ Correspondence should be addressed to M. Stephen Hodgart, s.hodgart@surrey.ac.uk
}

Received 6 July 2011; Revised 6 November 2011; Accepted 7 November 2011

Academic Editor: Simonetta Paloscia

Copyright ( 2011 M. Stephen Hodgart. This is an open access article distributed under the Creative Commons Attribution License, which permits unrestricted use, distribution, and reproduction in any medium, provided the original work is properly cited.

The upcoming Galileo global navigation satellite system has a design problem with the cosine-phased BOCc(15, 2.5) modulation of its Public Regulated Service (PRS A-code). This signal needs far more bandwidth than the available $40.92 \mathrm{MHz}$. The present signal and system specification cannot be expected to deliver design performance under practical operational conditions (noise, receiver phase distortion, and multipath). There would not have been this problem with sine-phased BOC(15, 2.5).

\section{Introduction}

The Galileo global navigation satellite system has been more than a decade in the design and planning. The first two IOV satellites have been launched and at time of writing (January 2012) are being tested. The next two IOV satellites and FOC satellites are under construction but at present there is nothing in space to allow for a full check of their navigational capability. As is well known at least four satellites have to be up and running to attempt a full navigational solution. It is at that point this paper anticipates that some inherent problems will be confirmed because of an inherent design problem (not to say fault).

It may seem surprising that after such a concerted effort and expert input that there could be any such a possibility. But if a system does not work properly, then it does not work. If the predictions of this paper turn out to be wrong, then there is of course no cause for concern. But if the predictions are right, then the reasons are fully explained here-as is the necessary correction.

The difficulty lies with the flag-ship PRS A-code (public regulated service): the wide-bandwidth signal transmitting on $\mathrm{E} 1 \mathrm{channel}$ (centred on $1575.42 \mathrm{MHz}$ ). It is predicted here that navigation receivers trying to track this signal and compute their location are likely to fail in any but the most ideal conditions. Low-strength signal (drop-outs), multipath, and all the contingent practical imperfections associated with real-world reception will make tracking and computations of the navigation solution unreliable. The same problem but less severe in its effect can be expected in reception of the other PRS -A code signal transmitting in the E6 channel centred on $1278.75 \mathrm{MHz}$.

There are hints that responsible engineers already recognise the problem to come but are understandably reluctant to say so explicitly — see, for example, recent conference papers hosted by ESTEC $[1,2]$. With the GATE test facility (Galileo Test Range) in Germany coming online experimental evidence ought to be forthcoming. These are however limited to a terrestrial test [3]. Such evidence may have featured in the technical papers most unfortunately withdrawn at the last minute by ESA from the European Navigation Conference held in October 2010 [4]. The date by which all four IOV (in orbit validation) will be in orbit and working has now slipped to some unadvertised time in 2012 [5]. It is to be noted however that these four satellites are not intended as truly experimental vehicles: they are supposed to be a committed part of the overall system. Therefore, assuming confirmation of the difficulties revealed in this paper, and if the necessary changes to the signal specification cannot be made after insertion in orbit, the IOV satellites will be transmitting a permanently deficient component of the system's transmissions as far as PRS is concerned. Likewise there will be trouble if the succeeding tranche of 14 "full-operational constellation" (FOC) satellites cannot be readily modified. The problem is a fundamental one. 


\section{Preview of the Problem}

The Galileo Signal Task Force (GSTF) appointed by the European Commission chose for its PRS A-code signal a BOC modulation [6]. The designation $\mathrm{BOC}$ is the binary offset carrier. In $\operatorname{BOCc}(15,2.5)$ or $\mathrm{BOC}_{\mathrm{cos}}(15,2.5)$, the " 15 " stands for $15 \times 1.023 \mathrm{MHz}$, the " 2.5 " for a $2.5 \times 1.023 \mathrm{MHz}$, and the " $c$ " or subscript "cos" indicates the choice of cosine phasing of the subcarrier relative to the phase of the code.

The problem is this: in order to work properly, a receiver of this BOCc $(15,2.5)$ ideally needs double the allocated channel bandwidth. Since there is no more available bandwidth, the only feasible alternative for a robust working system which will also achieve the expected performance is to change the modulation. The previous choice by the GSTF [7] was $\operatorname{BOC}(15,2.5)$ having the same rates of subcarrier and code but sine phasing would have worked satisfactorily, delivering the expected high precision within the available bandwidth.

The fact that a change of phase will alter the bandwidth requirements of an operational system by a ratio of $2: 1$ will seem as counter-intuitive to many technically minded readers as it must have been to the members of the GSTF (who designed the system). But BOC modulation makes for a complicated signal, and navigational requirements have a number of subtle aspects. It is understandable how the point seemingly was not appreciated in the overall signal design. But it is perhaps significant that Betz's original specification for BOC only ever envisaged sine phasing and that the USupgraded GPS adopts this phase only.

In this paper this key finding is given a relatively simple nonmathematical explanation and then demonstrated in a modification of the standard computer-generated multipath test. A new test criterion is offered and explained in order to justify the conclusion and completes the paper.

2.1. The Problem Begins with BOC. Ever since John Betz proposed BOC [8], it has received much technical attention on how to make it work properly. Unlike the heritage GPS system the introduction of a sub-carrier requires an optimised receiver to make a fine estimate of range to each satellite by tracking the sub-carrier component. The receiver must however resolve cycle ambiguities by independently making a coarse estimate of range from the code component only. In a maximum likelihood implementation there has to be a twostage process of range estimation in each channel.

In a standard correlating receiver the problem is exhibited as a multipeaked correlation with a need to track only on its primary peak. The coarse estimate is implied in the timing of the peak envelope of this correlation. The key requirement is that the coarse estimate must not be so coarse that it cannot resolve reliably the ambiguities in the fine estimate. This translates into a requirement that the main peak maintains a significant amplitude margin over adjacent secondary peaks.

It is here that Galileo's $\mathrm{BOC}_{\mathrm{cos}}(15,2.5)$ gets into difficulties. Cosine phasing generates a significant outer spectrumrequiring a much wider bandwidth than has been actually allocated to the channel. When a receiver of a cosine-phased BOC does not get to see this outer spectrum, then the accuracy of the fine estimate is not affected. No problem there.
The subtle difficulty is that the receiver needs to see this outer spectrum in order to make a good coarse estimate. An insufficient allocated channel bandwidth degrades the potential accuracy of a receiver's coarse estimate to the extent of the receiver being unable to correct reliably the ambiguities in the fine estimate. The combination of (i) high ratio $(6: 1)$ of subcarrier frequency to code rate (ii) relatively high subcarrier frequency, (iii) relatively low assigned bandwidth and (crucially), and (iv) cosine phasing puts the reliability of the two-stage process continually at risk for this particular BOC signal.

The risk can be assessed in a number of ways-in conditions of low input C/N0, phase distortion in the channel, and above all multipath.

The same difficulty is associated with the $\operatorname{BOCc}(10,5)$ signal-but less acutely_-see later comments.

2.2. Cosine Phasing Needs More Bandwidth. The bandwidth allocated to the E1/L1 channel for the test satellite GIOVE-B was $40.92=40 \times 1.023 \mathrm{MHz}$ - as confirmed by actual tests at Chilbolton [9]. It is also what is planned for the IOV satellites [10]. It is not enough for the cosine-phased option. The $\operatorname{BOCc}(15,2.5)$ signal needs a bandwidth allocation in the channel ideally double this amount-at $81.84=80 \times$ $1.023 \mathrm{MHz}$. As remarked already this assertion might seem intuitively wrong but it readily justified. The argument therefore is that insufficient bandwidth $\rightarrow$ loss of outer spectrum $\rightarrow$ degradation of coarse estimate $\rightarrow$ unreliable corrections of fine estimate ambiguities $\rightarrow$ failure of navigational solution.

2.3. Defining Failure. A navigational receiver may of course fail to work in all manner of ways. For avoidance of doubt failure means here the inability of the receiver to resolve the inherent ambiguities in range determination when tracking the sub-carrier for a fine estimate. Arbitrary unacceptable digital errors are the consequence imposed on a natural "analogue" error. These errors in range for $\operatorname{BOC}(15,2.5)$ are positive or negative integer multiples of $10 / 1.023 \mathrm{~m}$.

2.4. Sine Phasing Has Enough Bandwidth. The allocated channel bandwidth is sufficient for sine-phased $\operatorname{BOC}(15,2.5)$, that is, $\mathrm{BOC}_{\sin }(15,2.5)$ to work satisfactorily. The reason is simply that sine-phased BOC has no significant outer spectrum. Simulations run by the author predict that receiver operation with this phasing is not significantly degraded even if the signal is filtered to a bandwidth as low as 35 $\times 1.023 \mathrm{MHz}$. This modulation of sine-phased $\operatorname{BOC}(15,2.5)$ was of course the earlier choice by the GSTF before they finally decided on "cosine phasing."

The other PRS A-code signal $\mathrm{BOC}_{\mathrm{cos}}(10,5)$ centred on 1278.75 MHz has the same problem with cosine phasing but it is not so badly degraded. The specification for the E6 channel is again for a bandwidth of $40.92 \mathrm{MHz}$. But this modulation with its lower value of sub-carrier frequency and a higher code rate-the ratio is now $2: 1$ - has a greater separation of ambiguous fine estimates and is more capable of delivering a coarse estimate of sufficient accuracy. 


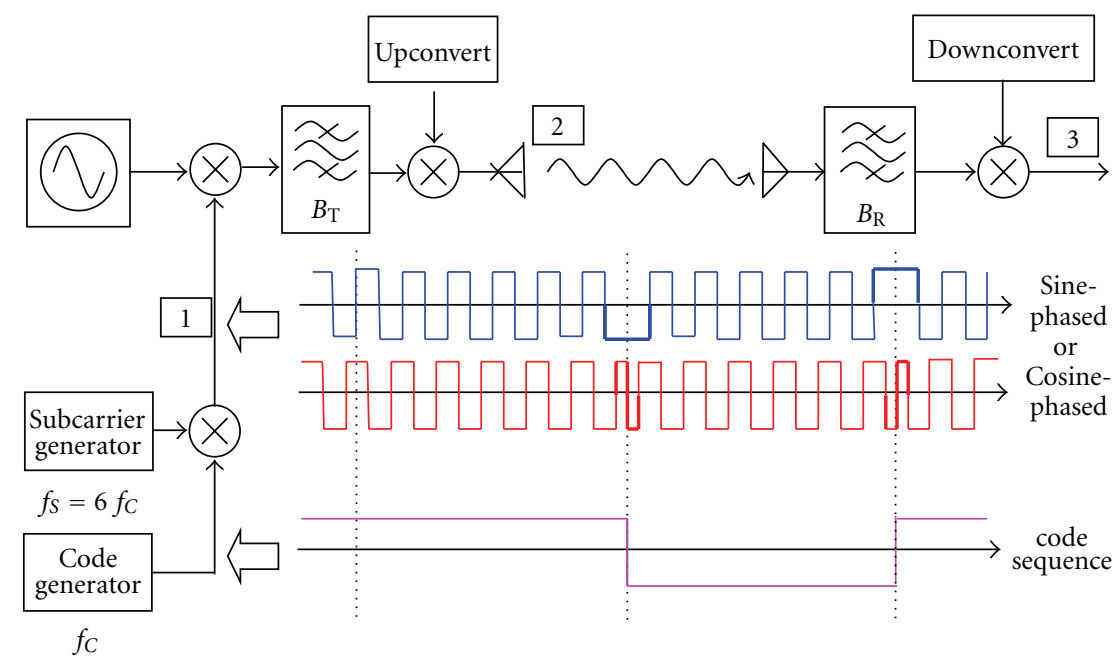

FIGURE 1: Simplified GNSS channel from transmitter to receiver transmitting two possible phases of sub-carrier to code for BOC(15, 2.5). Example code (magenta); cosine option (red); sine option (blue). Code rate $f_{C}=2.5 \times 1.023 \mathrm{MHz}$ sub-carrier frequency $f_{S}=15 \times$ $1.023 \mathrm{MHz}$.

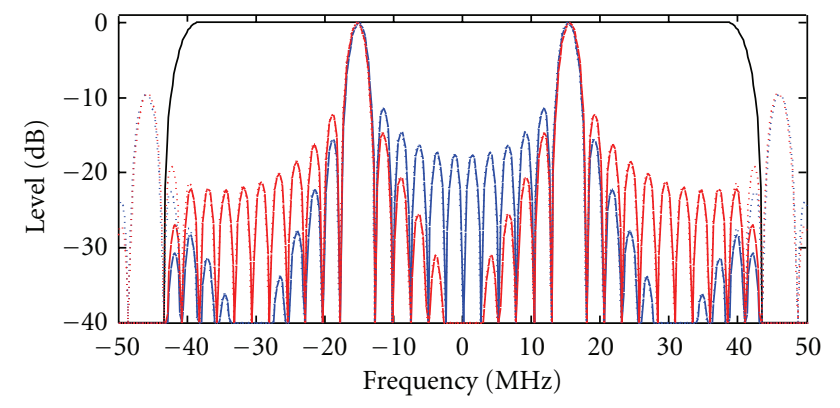

Figure 2: Ultra-wide TX filter (black) $B_{\mathrm{T}}=80 \times 1.023 \mathrm{MHz}$. Power spectra at [P2] (continuous red). Theoretical spectrum before TX filter (dotted red). Comparative filtered spectra for sine phasing (blue).

Focussing our attention on the $\operatorname{BOC}(15,2.5)$ signal the crucial point is to explain how a change from sine to cosine phasing-from the earlier sine option to the cosine option and no other change to the specification-should double the required bandwidth.

\section{Review of BOC Modulation}

Figure 1 shows a simplified schematic of a single GNSS channel from one satellite to ground and adopting BOC modulation. A continuous pseudorandom code sequence is characterised by a code rate $f_{\mathrm{C}}$ (whose reciprocal is a chip width). The code waveform multiplies onto a continuous sub-carrier of frequency $f_{\mathrm{S}}$ as at [P1] and then onto a radio carrier. It is well known that the BOC spectrum is split into an upper and lower sidebands (see also Figure 2) relative to the carrier frequency. Indeed that was the whole point of its design.

The BOC specification in general requires an integer number of half cycles of sub-carrier per chip. For BOC $(15$, $2.5)$ there is a $6: 1$ ratio of sub-carrier frequency to code rate and therefore 12 half cycles or alternating "subchips" of subcarrier assigned to each chip.

Before transmission the signal is bandpass filtered (assigned here bandwidth $B_{\mathrm{T}}$ ) and upconverted at point [P2]. After transmission over space a GNSS signal must be downconverted in the user's receiver. There is necessarily a frontend filter-assigned here bandwidth $B_{\mathrm{R}}$. A lowpass representation of the recovered signal in real time exists at point [P3].

3.1. Difference between Cosine and Sine Phasing. There is a very considerable difference in the detailed signal structure between sine phasing and cosine phasing. The difference is visualised in a postulated example sequence showing part or whole of four chips in sequence with two alternations of polarity.

3.1.1. Sine Phasing. The transitions in the sub-carrier coincide exactly with pseudorandom transitions in the code sequence. The schematic example shows the previous choice by the GSTF for the PRS signal which was $\mathrm{BOC}_{\text {sin }}(15,2.5)$.

3.1.2. Cosine Phasing. The transitions in the sub-carrier are offset by a quarter cycle-half a subchip width-relative to the code transitions. The schematic shows the final choice by the GSTF for the PRS signal which is $\mathrm{BOC}_{\cos }(15,2.5)$.

3.2. Why Cosine Phasing Needs More Bandwidth. So what difference does the choice of phase make? A great deal. One can see this immediately from closer inspection of the waveforms. From one code chip to the next there is a $50 \%$ chance of a polarity switch. It occurs when the succeeding code chip pseudorandomly changes phase. On this change of phase the cosine phasing creates a full cycle at twice the sub-carrier frequency (heavy red). This will be defined here as a double-frequency pulse (d.f.p) whose frequency is 


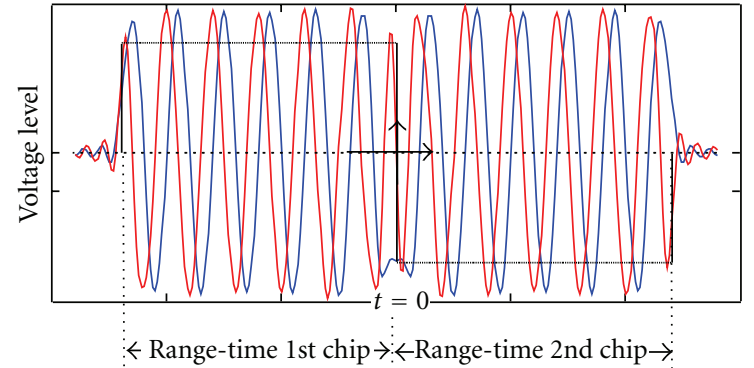

Figure 3: Two chips opposite polarity time domain response after RX filtering at [P3]. Ultra-wide bandwidth $B_{\mathrm{T}}=B_{\mathrm{R}}=80 \times 1.023=$ 81.84 MHz. Cosine optionred; sine optionblue.

$30.69=30 \times 1.023 \mathrm{MHz}$. But $\mathrm{BOC}_{\sin }(15,2.5)$ never generates this pulse. Instead, on a sign change there is generated a wider unipolar pulse-half a cycle at half the sub-carrier frequency (heavy blue).

3.2.1. Effect in the Frequency Domain. One can see the effect in the frequency domain. Figure 2 shows comparative plots of the ideal power spectra of the two transmissions using standard formulas available in the literature. For the cosine option (red) the pseudo-random sequence of doublefrequency pulses in the signal has given rise to a significant sideband content in the outer frequency regions. The sine option (blue) is quite different-it can be seen that the significant content lies inside the main lobes. The reason is simply because the pseudo-random transitional pulse is quite different-being a uni-polar pulse of half the sub-carrier frequency.

A linear-phase filter of any desired bandwidth can be assigned to a theoretical transmitter. The difference between full and dotted curves shows the effect of filtering. The black curve in Figure 2 depicts a TX filter whose bandwidth is exactly double the present assignment to the Galileo E1 band. With a standard raised cosine power response the filter rollsoff at $\pm 37.5 \times 1.023 \mathrm{MHz}$ and falls to zero at $\pm 42.5 \times$ $1.023 \mathrm{MHz}$. The $3 \mathrm{~dB}$ bandwidth $B_{\mathrm{T}}$ equals $80 \times 1.023 \mathrm{MHz}$. A matching filter is assigned to the receiver with the same $B_{\mathrm{R}}$ $=B_{\mathrm{T}}$.

3.2.2. Effect in the Time Domain. One can test for the presence of the double-frequency pulse on the filtered cosinephased BOC by computing the real-time signal at point [P3] of the system. Figure 3 shows one $\operatorname{BOC}(15,2.5)$ chip followed by another of opposite sign (the transition occurring at $t=0$ ). The six cycles of sub-carrier per code chip and the transitional d.f.p are clearly observable. The ultra-wide bandwidth therefore is adequate to maintain this key feature of cosinephased BOC.

\section{Available Bandwidth from Galileo}

But Galileo is not going to deliver such a wide bandwidth. The official bandwidth for the E1 channel is exactly half at $40 \times 1.023=40.92 \mathrm{MHz}$. The Chilbolton tests on GIOVE-B

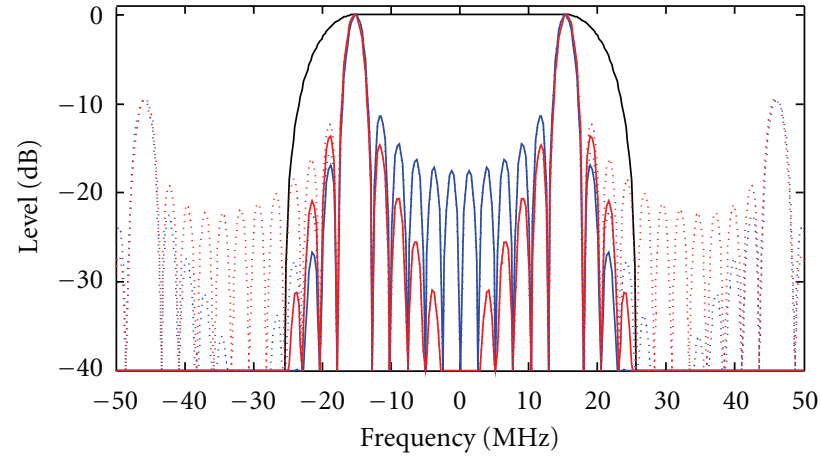

Figure 4: TX filter as GIOVE-B (black) $B_{\mathrm{T}}=40 \times 1.023 \mathrm{MHz}$. Power spectra at [P2] (continuous red). Theoretical spectrum before TX filter (dotted red). Comparative filtered spectra for sine phasing (blue).

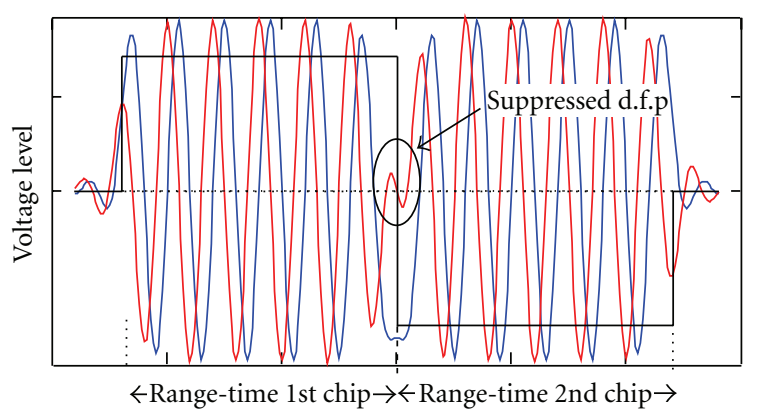

FIGURE 5: Time domain response at [P3]; sequential chips opposite polarity. Linear phase filters. TX filter as GIOVE-B. Matching filter in receiver. $B_{\mathrm{R}}=B_{\mathrm{T}}=40 \times 1.023 \mathrm{MHz}$. Cosine option red: sine option: blue.

measured the transmitted power spectrum of the $\mathrm{BOCc}(15$, 2.5) signal. The 1st, 2 nd, and 3rd outer side lobes are clearly discernible. There is no indication of any higher frequency sidelobes (which would have appeared above the noise floor). So these must have been filtered out. The GIOVE-B spectrum is presumed here to be representative of what is planned for the IOV series (and the FOC to follow).

In Figure 4 by adjusting a transmitter filter (black curve) to the theoretical spectrum (shown in dotted red) the continuous red curve closely reproduces the Chilbolton test measurements. Setting that filter to roll-off at $\pm f_{1}= \pm 15 \times$ $1.023 \mathrm{MHz}$ and cut-off at $\pm f_{2}= \pm 25 \times 1.023 \mathrm{MHz}$ sufficiently matches experiment: the specification matches the official $3 \mathrm{~dB}$ allocated bandwidth $B_{\mathrm{T}}=40 \times 1.023 \mathrm{MHz}$. We assume again a matching filter in the receiver time with $B_{\mathrm{R}}=$ $40 \times 1.023 \mathrm{MHz}$. In Figure 5 the resulting propagation of sine-phased BOC pulses on a change of code sign again proceeds smoothly although there is some distortion. The cosine-phased signal is however heavily distorted on the transition, and the transitional double pulse is largely suppressed.

These computer-generated tests from Figures $2-5$ demonstrate that the system needs double the presently assigned bandwidth at $B_{\mathrm{T}}=B_{\mathrm{R}}=81.84 \mathrm{MHz}$ in order to propagate 


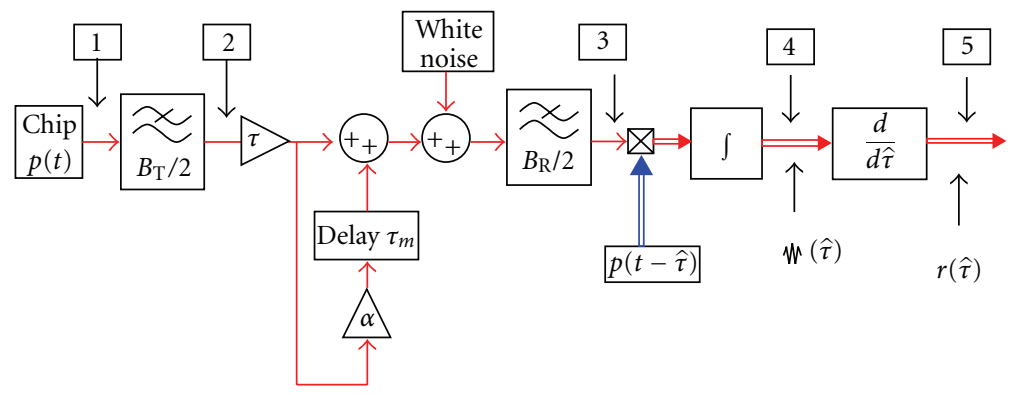

Figure 6: System modelling (base band) maximum likelihood (ML) receiver of BOC/PSK signal + multipath.

this double-frequency pulse. So in a preliminary summary, one has the following.

(1) Cosine-phased BOC has a significantly higher frequency content than sine-phased BOC because it pseudorandomly generates a double-frequency pulse (d.f.p).

(2) The practically available band-limited channel does not propagate this d.f.p.

4.1. Why More Bandwidth Matters. The key question is if failure to propagate this pulse should be important to the correct operation of a BOC receiver. The answer is that it is very important. The reason is not so much in the power but in the information that is now being lost. A navigation system is unlike a communication system in that the essential information is contained in the signal transitions not in the signal levels. The relative ability to locate transitions determines the ranging accuracy. Looking at Figure 5 and comparing with Figure 3 it might seem that the ranging accuracy from measurements of the multiple transitions in the subcarrier should be about the same in both cases. This expectation can be confirmed as far as the fine estimate is concerned.

But ranging information is also contained in the code transitions. These are what the heritage GPS depends on, and these transitions continue to provide the basis of the coarse estimate for BOC. In the case of cosine BOC doublefrequency pulse exists whenever there is a change of sign on a code transition. The effect of limited bandwidth which loses that pulse will be shown to degrade that coarse estimate. This degradation is what affects the two-level operation of a BOC receiver.

\section{BOC Correlating Receivers}

Recall that the aim of one GNSS receiver (channel) is to estimate the range to a transmitting satellite. A receiver does this by estimating the relative time of arrival of that signal and indirectly computing the propagation delay of that transmission.

5.1. General Principles. It is well known that BOC supports a large number of receiver implementations including one in which the sub-carrier is ignored and only the code component is tracked.

A BOC receiver in any configuration must deliver at least a low-level coarse estimate by tracking just the code component in the transmitted signal. In effect the receiver "sees" only the transitions in the code component of the signal and treats it like the ordinary BPSK transmission of the heritage GPS system. In order however to realise the potential accuracy of the BOC signal, a maximum likelihood receiver must generate a high-level fine estimate by tracking the sub-carrier component. But there are multiple solutions to this estimate-only one of which is correct. The multiple solutions are spaced at half a wavelength of the sub-carrier. The receiver must have therefore that other solution-the coarse estimate-in order to resolve this ambiguity. The coarse estimate-derived from the code transitions - must be available at all times assuming that the fine estimate is always liable to cycle slip from a valid to an invalid solution.

The coarse estimate must not however be too coarse. It needs to be sufficiently accurate to be able to distinguish reliably between the right solution from the adjacent wrong solutions by that fine estimate. The most accurate $\mathrm{BOC}$ receiver has to deliver two estimates continuously-the ambiguous fine estimate and an unambiguous coarse estimate where the latter is always "on standby" and is sufficiently accurate to resolve the ambiguities in the former.

5.2. Model System. A simplified model of a channel feeding a maximum likelihood BOC system-transmitter, channel, and receiver, is shown in Figure 6. Details of the coding structure are not relevant here.

The upconversion and downconversion process is simplified to a base-band representation. Carrier recovery is not the issue. The unfiltered chip input at [P1] in the transmitter is equivalently lowpass filtered at [P2]. The signal propagates over space after a delay $\tau$ to the distant receiver. The effect of multipath is modelled by adding/subtracting a single indirect path of relative amplitude $\alpha$ at the input after a local delay $\tau_{\mathrm{m}}$. If running a jitter and signal-to-noise analysis white noise can be added at this point before entering the receiver. The signal is now filtered again in the receiver to point [P3].

The filtered chip at point [P3] is multiplied by an unfiltered replica which embodies a trial delay $\hat{\tau}$. The product is integrated to an observable multipeaked correlation at point [P4] which is a function of trial delay $\hat{\tau}$ in the applied 


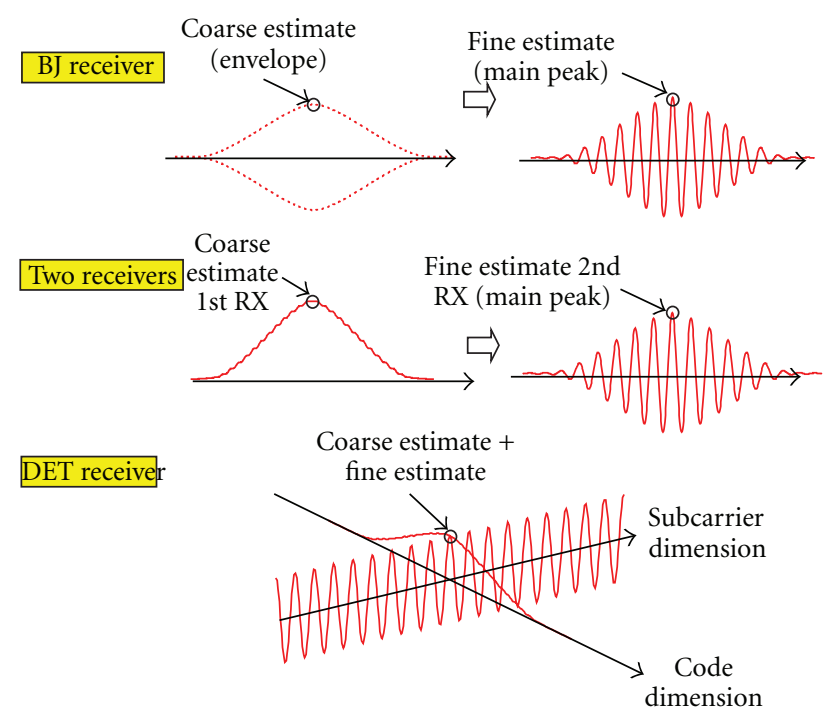

FIGURE 7: Schematic of correlation shapes in three basic types of optimal BOC receiver.

replica. The correlation function is effectively differentiated to a discriminant function $r(\hat{\tau})$ at point [P5] which looks for the main zero-crossing in the differentiated correlation.

It is to be understood that the receiver has to generate a vector of various trial values of $\hat{\tau}$ in order to discern sufficiently the shape of the correlation. At the very least there needs to be a "prompt," a "late," and an "early" estimate.

The presence of sub-carrier and the ambiguity is revealed in these multiple peaks (include negative). The separation of inner peaks (count both negative and positive) is half the period of the sub-carrier. Only tracking the centre peak gives the correct answer. A coarse estimate is not explicitly computed but is implicitly located in the $\Lambda$-shaped envelope of the actual correlation.

In the bump-jumping receiver [11] resolution of ambiguity depends therefore on measurement of relative amplitudes of adjacent peaks and "bump-jumping" the fine estimate forward or backwards as necessary in the direction of the peak with discernibly the greatest amplitude.

In an alternative two receiver or "BPSK-like" concept [12] an independent 1st receiver effectively generates an actual $\Lambda()$-shaped correlation from the code component onlyignoring the sub-carrier component. The coarse estimate is therefore explicitly determined. The fine estimate is then determined separately in a 2 nd receiver by correlating the input signal against a replica just as in the receiver of Figure 6. There is no "bump-jumping" as such. An overall control uses the coarse estimate to steer the fine estimate onto the correct peak of the correlation.

In yet another concept of double-estimation technique DET [13] the system of Figure 6 is generalised by splitting the reference replica into separate code and sub-carrier components with independently assigned trial delays controlled by independent loops. The system effectively creates a 2 -dimensional correlation within the receiver by separating code and sub-carrier component, varying their timing independently but interactively. The peak of a $\Lambda$-shaped correlation is tracked in the code dimension, and a continuous periodic correlation is tracked in the sub-carrier dimension. The coarse estimate from the former forces an integer correction of the fine estimate to a valid solution.

See Figure 7 for graphical depiction of these different systems. Where they all fail is if and when the measurementimplicit or explicit—of the coarse estimate is not sufficiently accurate to resolve the ambiguities in the fine estimate.

However it is derived that the coarse estimate will generally have a greater error than the fine estimate. But the relative error between the two cannot exceed a specific limit. The multiple solutions to the fine estimate are spaced at half a wavelength of the sub-carrier frequency. For any receiver the relative error between the coarse estimate and the correct fine estimate must not exceed quarter a wavelength $(\lambda / 4)$. For $\operatorname{BOC}(15,2.5)$ the solutions are spaced at $10 / 1.023 \mathrm{~m}$ and the relative error must not exceed $\pm \lambda / 4=5 / 1.023 \mathrm{~m}$. If it should go outside this limit, the receiver will fail.

The problem with band-limited cosine-phased BOC is that the relative error in the coarse estimate is much more likely to exceed this limit under representative worst case conditions.

We test this assertion on the model receiver implementing the $\mathrm{BJ}$ "bump-jumping" concept. However exactly the same general conclusions follow for the other two possible implementations. DET may give a better performance on initial acquisition and pull-in but cannot overcome this tracking problem. Early tests in simulations did not appreciate the fundamental difficulties described here [14].

It is immediately clear that if the effect of noise and distortion is to drive adjacent secondary peaks to an equal or greater amplitude than the primary peak, then eventual failure follows. This is more likely to happen with a flatter envelope of the correlation-since there will be less amplitude margin between primary and secondary peaks. It is even more likely to happen with a shifted envelope relative to the actual correlation.

The critical condition is depicted in Figure 8 with a relative shift by $\lambda / 4$. On this limit adjacent peaks to the correlation-one negative the other positive-will have exactly the same amplitude and it will not be possible to distinguish true from false solution.

If the relative error should exceed the limit then the receiver shifts to a semipermanent condition of false lock, since it now could be tracking the wrong peak in the multipeaked correlation.

\section{Effect of Limited Available Bandwidth}

6.1. Amplitude Distortion. The graph of Figure 9 depicts the rectified correlation at $[\mathrm{P} 4]$ for the system of Figure 6. Blue and red curves are for sine and cosine, respectively, of the rectified correlation. The dotted curves are the envelope $\Lambda()$ function which is computed by a standard technique involving a Hilbert Transform. The filters at both transmitter and receiver are both assumed to be linear phase. So there is only amplitude distortion.

We can define an amplitude margin as between the primary peak and the worst case adjacent secondary peak. In 


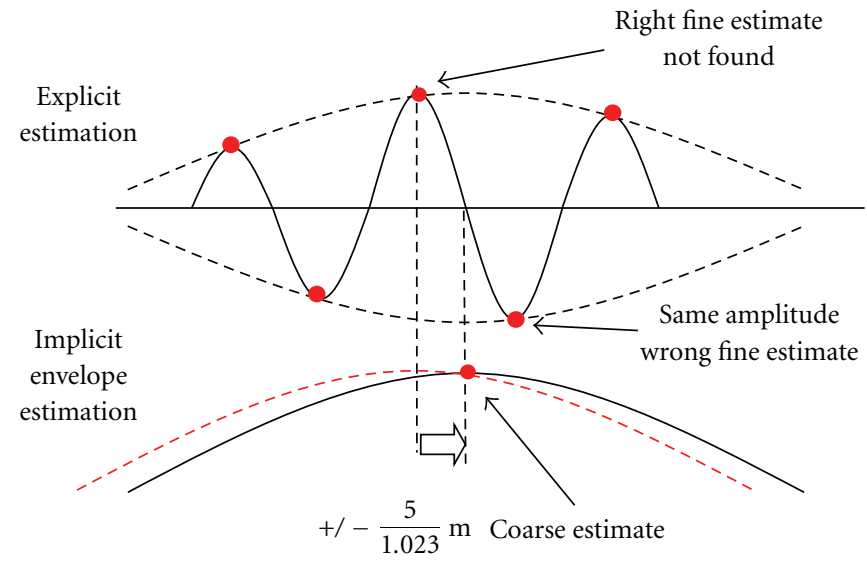

FIgURE 8: Limit of BJ operation: max error $\lambda / 4$ between explicit fine and implicit coarse estimate.

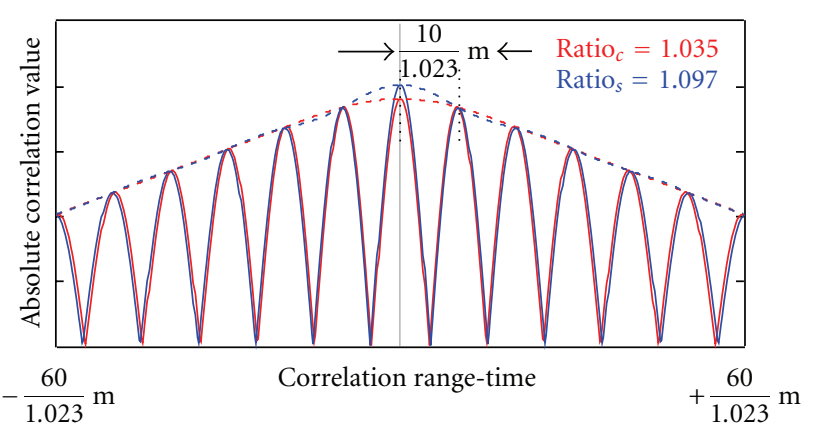

FIGURE 9: Rectified correlation. Linear phase TX filter $B_{\mathrm{T}}=40 \times$ 1.023 MHz as GIOVE-B. red: cosine option; blue: sine option. Linear phase matching filter is, $B_{\mathrm{R}}=B_{\mathrm{T}}$. Dotted linesenvelope.

a channel of infinite bandwidth, the correlations are much the same and the amplitude margin is essentially the same.

In the move from $\mathrm{BOC}_{\sin }(15,2.5)$ to $\mathrm{BOC}_{\mathrm{cos}}(15,2.5)$ assuming very wide-band theoretical filters with $B_{\mathrm{T}}=B_{\mathrm{R}}=$ $81.84 \mathrm{MHz}$, the amplitude margin is slightly reduced from adequate $11.0 \%$ to $9.1 \%$, respectively. But for the practical bandwidth available and matching filter $B_{\mathrm{T}}=B_{\mathrm{R}}=$ $40.92 \mathrm{MHz}$, the margins are drastically reduced from $9.7 \%$ to $3.5 \%$. The respective correlations are shown in Figure 9.

For the same value $B_{\mathrm{T}}$ and brick-wall filtering in the receiver with a bandwidth $B_{\mathrm{R}}=35 \times 1.023 \mathrm{MHz}$, it can be shown that the margins are $9.3 \%$ and $2.7 \%$, respectively. Filtering has flattened the envelope for the cosine option. Interestingly it has no significant effect on the sine option. Perhaps counter-intuitively even quite severe band limiting is seen to sharpen up the correlation. An even tighter brick-wall filter which is encroaching on the main lobes can be shown to improve the margin. Practical band limiting seriously reduces the margin for the cosine option but not the sine option.

6.2. Phase Distortion. A practical system introduces phase distortion as well-in both transmitter and receiver unless deliberately compensated. The effect is modelled here by making the input filter to the receiver a standard resonator type filter with a sharp cut-off outside the passband. The

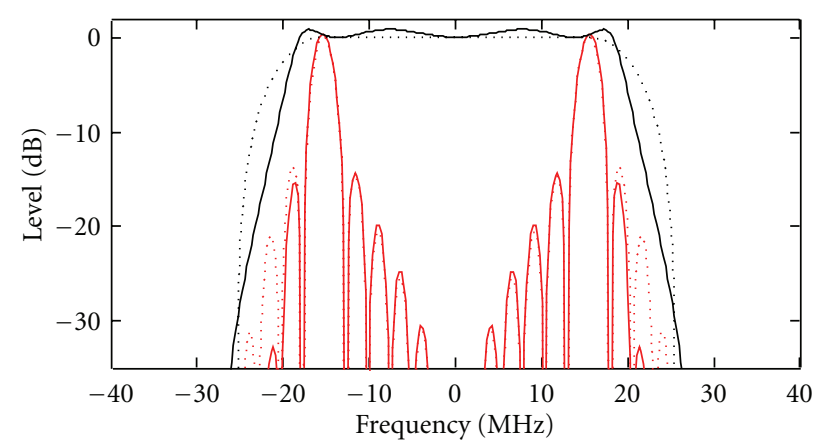

FIgure 10: Phase distortion. Rectified correlation. Linear phase TX filter $B_{\mathrm{T}}=40 \times 1.023 \mathrm{MHz}$ as GIOVE-B. Red: cosine option; Blue: sine option. Cauer filter receiver is $B_{\mathrm{R}}=35.5 \times 1.023 \mathrm{MHz}$. Dotted lines: envelope.

same linear phase filter is assumed in the transmitter (dotted black). Figure 10 computes the effect of a front end RX filter with a standard elliptic-function (Cauer) response (continuous black). It is characterised here with a $10 \%$ pass band ripple and two notch zero response points outside the pass band (spaced 1.5 times the unity gain bandwidth edge).

The theoretical test is to maintain the same proportional shape to the receiver filter while varying its bandwidth. With phase distortion not only is the peak of the envelope flattened but the peak of the envelope function $\Lambda()$ is displaced relative to the peaks in the actual correlation. The resulting effect on the correlation is shown in Figure 11.

The combination of these two effects on the cosine option is seen to identify a critical bandwidth $B_{\mathrm{R}}=35.5 \times$ $1.023 \mathrm{MHz}$ where the amplitude margin is reduced to zero. At this critical point the peak of the envelope has been displaced relative to the primary peak by that critical amount of $5 / 1.023 \mathrm{~m}$. At this critical bandwidth a BOC receiver of the cosine option must fail because the coarse estimate is unable to distinguish the valid fine estimate from an invalid adjacent estimate located 10/1.023 $\mathrm{m}$ away. Yet the sine option (in blue) continues to have a significant margin $(6.4 \%)$. The problem here is a combination of both flattening and relative 


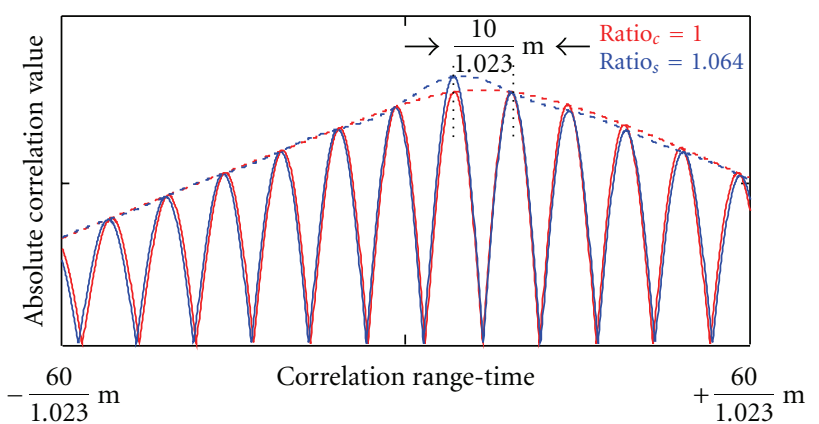

FIgUre 11: Phase distortion. Rectified correlation. Linear phase TX filter $B_{\mathrm{T}}=40 \times 1.023 \mathrm{MHz}$ as GIOVE-B. Red: cosine option; blue: sine option. Cauer filter receiver is $B_{\mathrm{R}}=35.5 \times 1.023 \mathrm{MHz}$. Dotted lines: envelope.

shifting of the envelope to the correlation. Yet the sine option is not significantly affected in a comparative test.

In principle phase compensation can be implemented in the receiver-as for the transmitter. But effective control and implementation of appropriate compensation in the receiver is a complex matter and may need to be adaptive. Automatically the receiver is not robust.

The point is the following

(1) the band-limited cosine option is far more sensitive to phase distortion than the sine option,

(2) without specific compensation a receiver of $\operatorname{BOCc}(15,2.5)$ will not work.

The transmitters for the Galileo satellites are constrained to a rigorous specification on linearity. Necessarily there must be less control over the receiver design.

6.3. Multipath Distortion. Multipath vector adds one or more extraneous signals to the main path. Adaptive compensation against multi-path is a complication which it is assumed here that the BOC receiver will not have implemented. Accordingly it is crucial to test what is the immediate effect of multipath on an unadapted receiver.

A standard test-following the GSTF (Galileo Signal Task Force) [7] —is to add or subtract a half amplitude single additional path to or from the main path, respectively, and compute the effect as a function of the relative delay $\tau_{\mathrm{m}}$ in that single path. The effect of this standard test is to introduce a time bias error in the location of the primary peak (and all the other peaks) of the correlation function. The effect of multi-path is also to introduce a time bias error to the peak of the envelope function $\Lambda()$. It can also greatly alter the shape of that envelope-although that effect is not considered here.

These bias errors generally are different: multipath therefore forces a relative error or relative bias between the coarse and the fine estimates. If this relative error exceeds the $\pm \lambda / 4$ limit, then receiver operation must fail. It is this crucial aspect of receiver operation which the GSTF could not have tested. If they had, they would have seen the problem.

We introduce therefore a new kind of test of BOC performance-one which may not have previously been

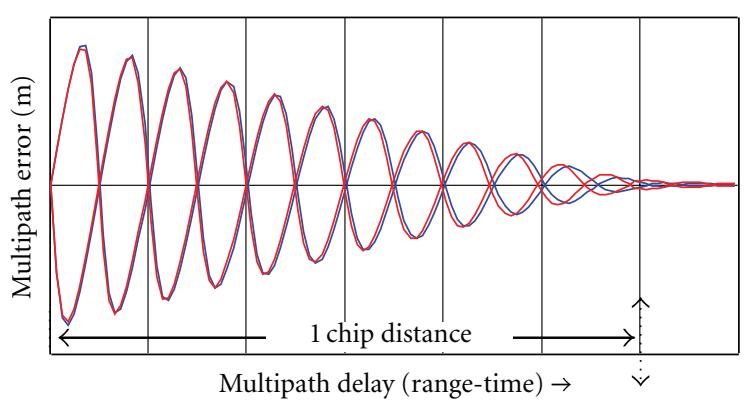

FIgure 12: Absolute multi-path error in main peak (fine estimate). Rectified correlation. Linear phase TX filter $B_{\mathrm{T}}=40 \times 1.023 \mathrm{MHz}$ as GIOVE-B. Red: cosine option; blue: sine option. Matching filter is $B_{\mathrm{R}}=B_{\mathrm{T}}$.

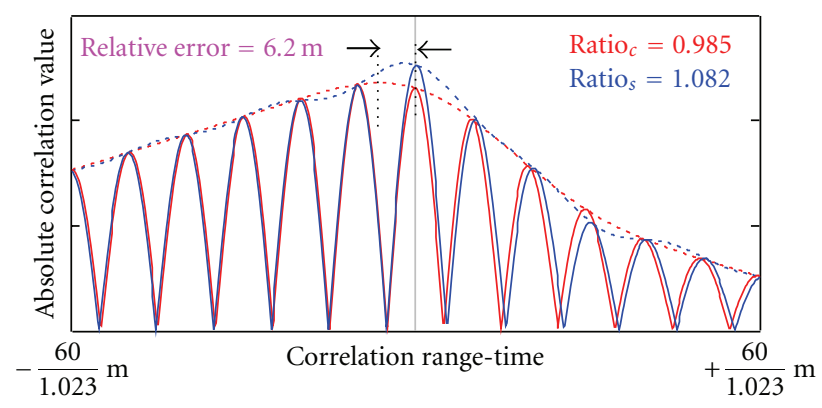

FIGURE 13: Worst case rectified correlation distorted by multi-path. Linear phase TX filter $B_{\mathrm{T}}=40 \times 1.023 \mathrm{MHz}$ as GIOVE-B. Red: cosine option; blue: sine option. Matching filter is $B_{\mathrm{R}}=\mathrm{B}_{\mathrm{T}}$. Half amplitude m.p delay $\tau_{m}=3 / 12$ of chip width. Dotted is envelope.

identified in the literature. Instead of just testing for the absolute shift of the correlation peak-as in the usual testwe now test for the relative shift between the correlation primary peak and its envelope. This test provides an immediate confirmation of the basic problem.

6.3.1. The Fine Estimate. Figure 12 shows the effect of multipath error on the fine estimate by a receiver of $\operatorname{BOC}(15,2.5)$. In this computer test the practical matching filter is again assumed. The effect of multipath on the timing of the main correlation peak and therefore the fine estimate is much the same for either cosine or sine phasing. We replicate here the published results of the GSTF as in [7]. The bias error varies cyclically with increasing multi-path delay. The bias error does not exceed a peak of around $\pm 1.8 \mathrm{~m}$. There is no important difference between the two options as far as the fine estimate is concerned.

6.3.2. The Coarse Estimate. The effect of multi-path on the coarse estimate is however very different, being much worse in the case of cosine phasing. An example is shown in Figure 13 where the half-amplitude multi-path has been assigned a delay of $3 / 12$ of a chip width. In this worst case value there is no significant error in the fine estimate but the coarse estimate is displaced by $6.2 \mathrm{~m}$. This is more than a quarter wavelength of the sub-carrier. The primary peak now has less 




Figure 14: Relative multi-path error between fine and coarse range estimate. Linear phase TX filter $B_{\mathrm{T}}=40 \times 1.023 \mathrm{MHz}$ as GIOVE-B. Red: cosine option; blue: sine option. Matching filter is $B_{\mathrm{R}}=B_{\mathrm{T}}$.

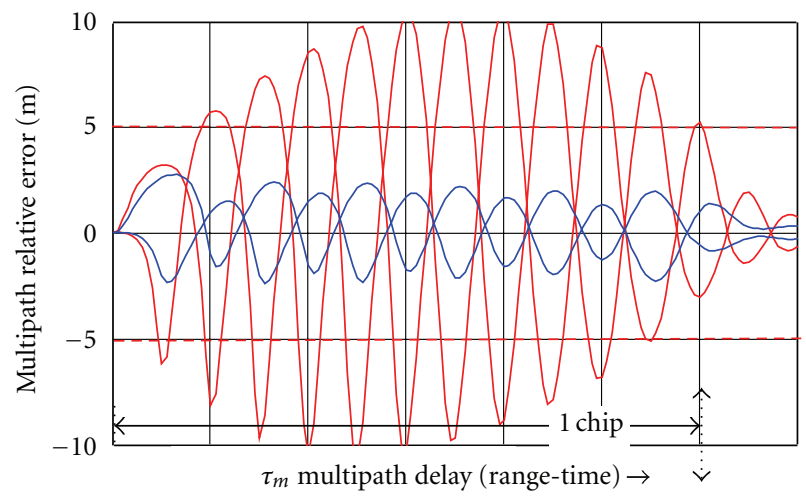

FIGURE 15: Relative multipath error between fine and coarse range estimate. Linear phase TX filter $B_{\mathrm{T}}=40 \times 1.023 \mathrm{MHz}$ as GIOVE- B. Red: cosine option; blue: sine option. Brickwall filter in RX is $B_{\mathrm{R}}=$ $35 \times 1.023 \mathrm{MHz}$.

amplitude than an adjacent secondary peak (the margin is now negative). The receiver will fail if this condition persists. Yet the sine phasing option is not nearly so badly affectedand importantly the main peak maintains the vital (positive) amplitude margin.

6.4. Relative Multipath Error. A computer search now implements the proposed new test: of the relative error as between correct peak of the correlation function and peak of its envelope over a range of delay times $\tau_{\mathrm{m}}$ (up to $120 / 1.023 \mathrm{~m}$ ). As shown in Figure 14 the peak relative error (in red) can rise to around three times that of the sine option (blue) and often exceeds the critical limit of $\pm 5 / 1.023 \mathrm{~m}$.

On this limit adjacent peaks (include both signs) of the actual correlation are the same amplitude and it is not possible to tell true from false. Over this limit and the receiver must slip into a condition of false lock and not recover. There are multiple opportunities for cycle slip — a condition which phase distortion, electrical noise, and other system errors can only exacerbate-to bring this about.

The deviations of the peak of the envelope for the sine option are clearly much less and stay well within limits.

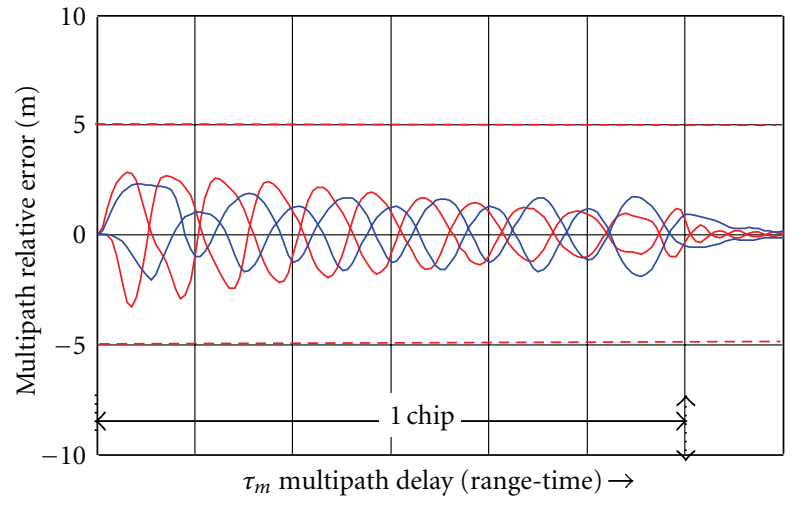

FIGURE 16: Relative multi-path error between fine and coarse range estimate. Ultra-wide TX bandwidth linear phase. Red: cosine option blue: sine option. Matching filter in receiver is $B_{\mathrm{T}}=B_{\mathrm{R}}=80 \times$ $1.023 \mathrm{MHz}$.

The extent of multi-path error for the brick wall filter is shown in Figure 15. A bandwidth of $35 \times 1.023 \mathrm{MHz}$ can be shown to cause no problem for the sine option but is a clear disaster for the cosine option. As can be seen the relative error over the range of delays generally exceeds the limit of $\pm 5 / 1.023 \mathrm{~m}$ - and can exceed $\pm 10 / 1.023 \mathrm{~m}$. Yet there is no significant worsening of multi-path error for the sine option.

To make the point that the problem is due to an insufficient bandwidth, we run an identical test assuming now that (somehow) the very much wider bandwidth could be allocated to the receiver and the transmitter where $B_{\mathrm{R}}=B_{\mathrm{T}}=$ $80 \times 1.023 \mathrm{MHz}$ (twice the Galileo allocation). The resulting plot as in Figure 16 computes the relative multi-path error. As can be seen the cosine option is now much the same as the sine option-although both are showing acceptably low error from multipath. But it needed this very large $B_{\mathrm{R}}=B_{\mathrm{T}}$ bandwidth in both transmitter and receiver. This final result confirms the source of the problem.

$B O C_{\cos }(10,5)$. Repeating the multi-path test for $\mathrm{BOC}_{\mathrm{cos}}(10$, 5 ) shows that the cosine option is again worse off although it is not so drastically affected. Again there is no significant difference in the effect of multi-path on the error to the fine estimate between the two options. It makes no essential difference which phase is adopted when tracking on the main central peak to the correlation. The critical allowed relative error for the difference between coarse estimate and fine estimate is now $\pm 7.5 \mathrm{~m}$. According to [10] the same bandwidth of $40 \times 1.023 \mathrm{MHz}$ has been assigned for E6 channel containing the $\mathrm{BOC}_{\mathrm{cos}}(10,5)$. One assumes therefore the same roll-off in the transmitter filter from $\pm 15 \times 1.023 \mathrm{MHz}$ and cut-off at $\pm 25 \times 1.023 \mathrm{MHz}$. If the same matching filter is assumed in the receiver, then the error in the coarse estimate is not significantly different as between cosine and sine options.

The cosine option is however degraded by a brick wall filter. The assumed receiver brick wall filter now has $B_{R}=30 \times$ $1.023 \mathrm{MHz}$ which bandwidth is just sufficient to include the main lobes of this transmission. 
Computer tests show that there are significant ranges of multi-path delay for which the relative error breaches the limit. For the sine-phased option it stays well within limits.

\section{Summary and Conclusions}

The conclusion to be drawn from this paper is that the PRS A code signal for E1 as presently specified is not going to work as expected and specified. What undermines this $\mathrm{BOC}_{\cos }(15$, 2.5) signal are factors no one of which is wrong individually but in combination will lead to indifferent operational performance if not outright failure. These factors are (i) high ratio of sub-carrier frequency to code rate, (ii) relatively high sub-carrier frequency, (iii) relatively low assigned bandwidth in the transmitter and receiver, and (iv) cosine phasing. Individually these conditions may be acceptable but not all in combination.

It is readily confirmed that the error due to multi-path in making the fine estimate is about the same for either cosine or sine phasing.

But BOC needs other estimates for a receiver to do its work properly - the coarse estimate derived from transitions in the code component. It must have this in order to resolve the ambiguities in the fine estimate. This is where the cosine option comes unstuck. It has been shown that the accurate determination of these transitions in the code depends on the propagation of double-frequency pulse (d.f.p) needing that much wider bandwidth.

The inability to transmit and receive the critical high-frequency content of the cosine phased signal degrades the twolevel BOC receiver for the reasons stated and explained. This fact has been demonstrated here most simply by introducing multi-path into a test calculation according to a standard test model. Deeper analysis will show a corresponding increased vulnerability to electrical noise in critical conditions of low $\mathrm{C} / \mathrm{N} 0$ and high loop bandwidth.

The source of the very great difference in bandwidth dependence is located in every chip transition when the pseudorandom code sequence changes phase. For the cosine option a bi-polar pulse is created which is double the sub-carrier frequency. For $\operatorname{BOCc}(15,2.5)$ a.k.a $\operatorname{BOC}_{\cos }(15,2.5)$ the frequency of this pulse is $30 \times 1.023=30.69 \mathrm{MHz}$. The requirement of this d.f.p has been shown to push up the inherent bandwidth - when doubled-up to allow for upper and lower sidebands-from 40.92 ideally to $81.84 \mathrm{MHz}$. Galileo is not going to provide this higher value of bandwidth. The available bandwidth will not be able to propagate this key signal element and deliver the much-vaunted accuracy of this new global navigation satellite system using PRS.

\section{Acknowledgments}

All information presented here comes from public sources. The deductions and conclusions are entirely the responsibility of the author. None of the views presented in this paper should be attributed or taken to represent the views individually or collectively of Surrey Space Centre, University of Surrey, or any associated company, or their representatives.
The work was done without financial support. The author is grateful for useful comments from the anonymous referee which have been incorporated this paper.

\section{References}

[1] N. Martin and H. Guichon, "BOC ambiguity resolution aided by virtual sub-carrier tracking for multi-path navigation," in Proceedings of the 4th European Workshop on GNSS Signals and Signal Processing, December 2009.

[2] H. Guichon, N. Martin, and M. Crisci, "Acquisition of BOC signal in presence of multipath," in Proceedings of the 5th Esa Workshop on Satellite Navigation Technologies (NAVITEC '10), December 2010.

[3] "Galileo Test Environment Open for Business," GPS world, February 2011.

[4] "Galileo ESA pulls sensitive presentations from conference," GPS World, October 2010.

[5] Galileo Edges Towards the Launch Pad, BBC News, 2010.

[6] J. Avila-Rodriguez, G. W. Hein, S. Wallner et al., "The MBOC modulation-a final touch for the Galileo Frequency and Signal Plan," in Proceedings of the 20th International Technical Meeting of the Satellite Division of the Institute of Navigation (ION GNSS '07), October 2007.

[7] G. Hein, M. Irsigler, J. A. Rodriguez, and T. Pany, "Performance of Galileo L1 signal candidates," in Proceedings of European Navigation Conference GNSS, May 2004.

[8] J. Betz, "The offset carrier modulation for GPS modernization," in Proceedings of the ION Technical Meeting, pp. 639648, Cambridge, Mass, USA, June 1999.

[9] G. Gatti, M. Falcone, V. Alpe et al., "Giove-B chilbolton inorbit test initial results from the second Galileo satellite," in Proceedings of the 21th International Technical Meeting of the Satellite Division of the Institute of Navigation (ION GNSS '08), pp. 30-35, October 2008.

[10] G. T. A. Burbidge, G. J. Robertson, T. J. Watson, and C. H. Mathew, "Galileo payload performance and status update," in Proceedings of the International Global Navigation Satellite Systems Society (IGNSS '09), Queensland, Australia, December 2009.

[11] P. Fine and W. Wilson, "Tracking algorithm for GPS offset carrier signals," in Proceedings of the National Technical Meeting of the Institute of Navigation NTM, The Institute of Navigation, San Diego, Calif, USA, January 1999.

[12] N. Martin, H. Guichon, M. Revol, M. Hollreiser, and M. Crisci, "Acquisition of the PRS BOC $(15,2.5)$ signal in presence of multi-path," in Proceedings of the 4th Esa Workshop on Satellite Navigation Technologies (NAVITEC '08), December 2008.

[13] S. Hodgart, P. D. Blunt, and M. Unwin, "Double estimator a new receiver principle for tracking BOC signals," in Proceedings of the 21th International Technical Meeting of the Satellite Division of the Institute of Navigation (ION GNSS '08), 2008.

[14] P. Blunt, R. Weiler, S. Hodgart, and M. Unwin, "Demonstration of $\operatorname{BOC}(15,2.5)$ acquisition and tracking with a prototype hardware receiver," in Proceedings of the European Navigation Conference, Geneva, Switzerland, May 2007. 

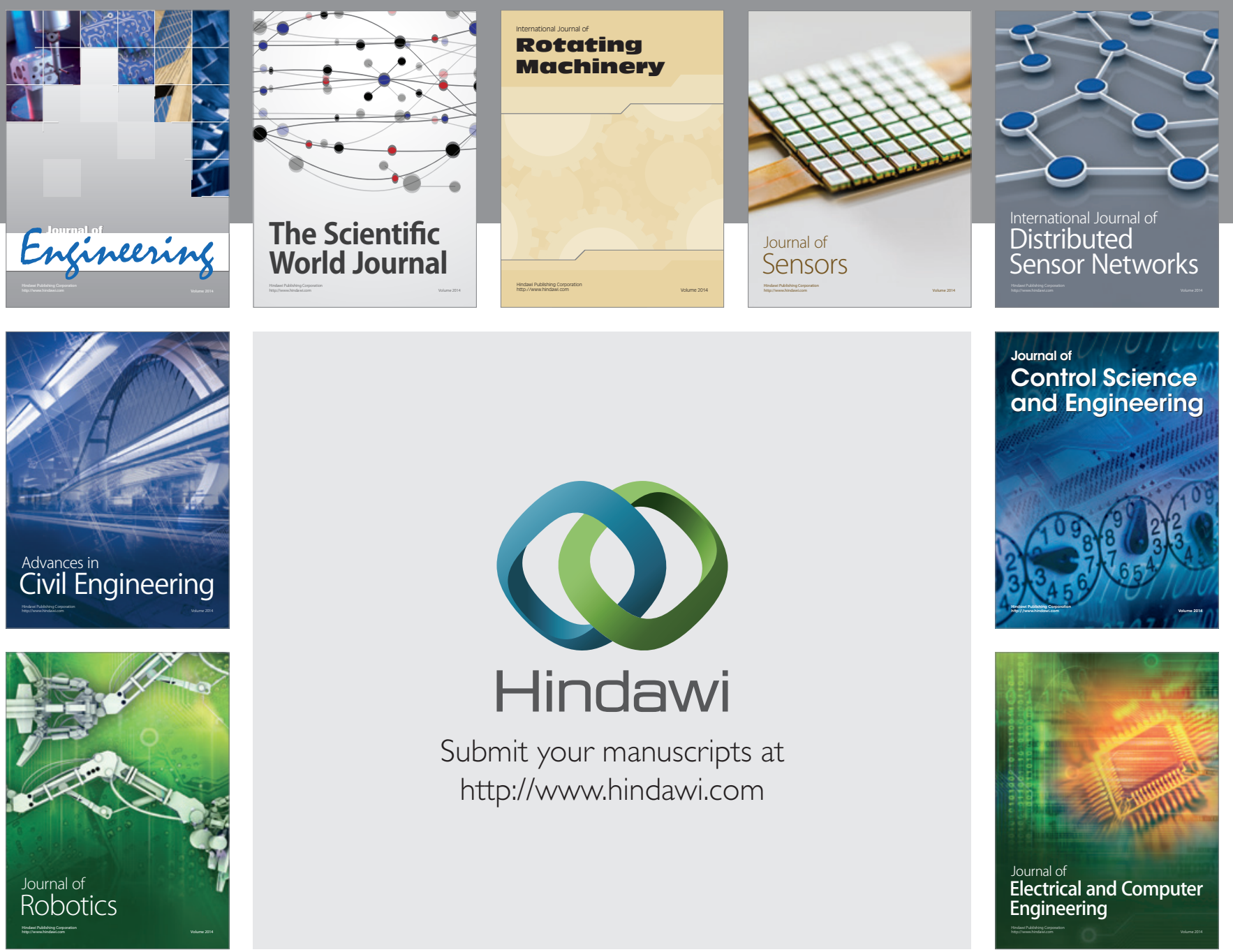

Submit your manuscripts at

http://www.hindawi.com
